\title{
Iron Deficiency Anaemia and Cancer
}

\author{
Sonia Bouri', John P Martin'
}

'Charing cross hospital, Imperial College London

\section{Article Info}

\section{Article Notes}

Received: January 29, 2018

Accepted: August 16, 2019

\section{${ }^{*}$ Correspondence:}

Dr. John P Martin, Charing cross hospital, Imperial College London; E-mail: johnmartin3@nhs.net.

(c) 2019 Martin JP. This article is distributed under the terms of the Creative Commons Attribution 4.0 International License.

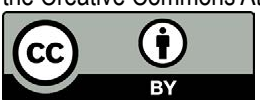

\section{Keywords}

Iron Deficiency Anaemia

Ferritin

Colorectal Cancer

Gastric Cancer

Colonoscopy

Gastroscopy

\section{ABSTRACT}

Iron deficiency anaemia (IDA) is a common condition. The finding of IDA should be given urgent attention as $8-15 \%$ of patients will be diagnosed with a gastrointestinal cancer and patients requiring urgent investigation should be identified. IDA is defined as a haemoglobin below the normal range with iron studies that indicate the presence of iron deficiency. This article will discuss the causes of IDA, interpretation of iron studies, and initial investigations for IDA. In the presence of infection or inflammation, the ferritin can be up to $100 \mu \mathrm{g} / \mathrm{l}$ even in the presence of IDA and these patients should be considered as having IDA if the other iron studies are supportive. Patients of all ages with IDA should have urinalysis and a Coeliac screen. Patients above 60 years and symptomatic patients of any age should be offered a colonoscopy and gastroscopy within 2 weeks. Men and women younger than 60 years, who are asymptomatic can have routine colonoscopy and gastroscopy. Pre-menopausal women should undergo the relevant endoscopy if they have symptoms or a family history of cancer. If initial bi-directional endoscopies do not show cancer, cancer should be considered in patients who do not respond to iron supplementation, which can indicate ongoing blood loss from the small bowel, or from extragastrointestinal sources.

\section{Text}

Iron deficiency anaemia (IDA) is a haemoglobin $(\mathrm{Hb})$ below the normal range of the laboratory performing the test in the presence of supportive iron studies ${ }^{1}$.

IDA occurs in $2-5 \%$ of men and post-menopausal women ${ }^{1}$. It is important to distinguish IDA from other causes of anaemia, as 8-15\% will be diagnosed with a gastrointestinal (GI) cancer and need urgent investigation ${ }^{1,2}$.

\section{Interpretation of blood tests}

In IDA, the mean cell volume and mean cell haemoglobin are reduced, however, they can also be reduced in anaemia of chronic disease (ACD), haemoglobinopathies and sideroblastic anaemia. In the presence of anaemia, the B12, folate and ferritin should be checked. A high red cell distribution width occurs in IDA, B12 deficiency and folate deficiency.

Ferritin is the best test for iron deficiency. A ferritin below the normal range alone is diagnostic of IDA. If infection, chronic inflammation or malignancy co-exists with IDA, the ferritin rises as an acute phase protein and the ferritin can be up to $100 \mu \mathrm{g} / \mathrm{l}$ (normal range $20-200 \mu \mathrm{g} / \mathrm{l}$ in our institution), even in the presence of iron deficiency. Therefore, further iron studies can help distinguish the cause of IDA in these cases. Iron studies include ferritin, serum iron, transferrin saturation, and total iron binding capacity or transferrin. 
Table 1. Iron studies patterns in IDA and anaemia of Chronic disease

\begin{tabular}{|l|c|c|c|}
\hline & Iron Deficiency & Anaemia of chronic disease & Iron deficiency and inflammation \\
\hline Ferritin & Low & Normal or High & Normal (up to 100 $\mu$ /I) \\
\hline Iron & Low & Low & Low \\
\hline TIBC/Transferrin & High & Low & Low or normal \\
\hline Transferrin Saturation & Low & Low & Low \\
\hline
\end{tabular}

Characteristic changes in iron studies which indicate IDA are shown in Table $1^{3}$. In IDA, there is a low transferrin saturation, ferritin and serum iron; and a high transferrin. Conversely, in ACD, the ferritin is high, due to an upregulation of hepcidin via IL- 6 which leads to reduced iron release from ferritin stores ${ }^{4-7}$, resulting in a low iron, low transferrin saturations and a low transferrin (Table 1). If ACD co-exists with IDA, it is widely accepted that a ferritin of greater than $100 \mu \mathrm{g} / \mathrm{l}$ can quite reliably rule out iron deficiency ${ }^{2}$, however, an international expert consensus has highlighted that for patients with chronic diseases such as chronic heart failure, chronic kidney disease and inflammatory bowel disease, the ferritin can be as high as $300 \mu \mathrm{g} / \mathrm{l}$ in the presence of confirmed iron deficiency, therefore in these cases, a transferrin saturation should be checked to confirm iron deficiency, with a transferrin saturation $<20 \%$ suggesting possible iron deficiency ${ }^{16}$.

\section{Causes}

IDA can be caused by blood loss from the GI tract including, ulcers and vascular abnormalities; $5-10 \%$ are caused by colonic cancer, $5 \%$ from gastric cancer and $1-2 \%$ from oesophageal cancer. Other causes include malabsorption, for example Coeliac disease helicobacter pylori infection and autoimmune gastritis; or inadequate dietary intake ${ }^{1}$. The commonest cause in pre-menopausal women is menstrual blood loss.

\section{History and examination}

A thorough history should include history of blood donation, dietary intake, visible blood loss, family history of haemoglobinopathies, drug history and menstrual history. Examination should check for abdominal masses and cutaneous signs of Peutz Jeghers or hereditary haemorrhagic telangiectasia.

\section{Investigations}

All IDA should be investigated ${ }^{1}$; every patient should be screened for Coeliac disease (prevalence is $0.5-1 \%$ ) and urinalysis, as $1 \%$ of IDA is due to a renal tract malignancy and one third of patients with renal cell carcinoma have IDA $^{8}$.

The NICE guidelines ${ }^{10}$ for suspected colorectal cancer were updated in 2017 to take into account the usefulness of the new fecal immunochemical test (FIT). They recommend that all patients over 60 years old with IDA should be referred via the two-week wait ( $2 \mathrm{WW}$ ) pathway and should undergo urgent bidirectional endoscopies. The $2 \mathrm{WW}$ pathway is also triggered at any age if there is IDA with rectal bleeding, weight loss or any abdominal or rectal masses.

For patients with IDA who do not meet referral criteria, the new recommendation, 2017, is to investigate using FIT at a threshold of 10 micrograms per gram of faeces ${ }^{10,15}$. This means that patients under 60 with IDA but no rectal bleeding, masses or weight loss should undergo a FIT. A positive test should trigger investigation via a $2 \mathrm{WW}$ referral. The FIT test which has been developed as a more accurate alternative to the previous guaiac based fecal occult blood test has been shown to have a good sensitivity in studies ${ }^{15}$, however it is in the early stages of use and both the awareness about the inclusion of this test in the updated NICE guidelines ${ }^{17}$ and the acceptability of this test in a non-screening population may be low. Furthermore, patients who do not strictly meet a $2 \mathrm{WW}$ referral criteria may still be deemed by the clinician's judgement to need endoscopic evaluation at an urgency determined by the patient's age, and the duration and degree of anaemia ${ }^{1,3}$. For example, a 55 year old male with IDA and a change in bowel habit, may be investigated endoscopically rather than with FIT. Patients who do not meet the referral criteria, but have symptoms of upper or lower GI disease or a strong family history of colon cancer should also be investigated endoscopically ${ }^{1}$. Menstruating women who are asymptomatic and do not have a strong family history, may not require a FIT depending on the duration and severity of the anaemia as menstrual blood loss is a common cause.

In the presence of a low ferritin, without anaemia, there is no consensus on which patients to investigate as the prevalence of cancer in this group is low. When selectively looking at men and post-menopausal women, the prevalence of cancer is $0.9 \%$ compared to $0 \%$ amongst pre-menopausal women, therefore it is suggested that bidirectional endoscopies are performed in patients who are over 50 years old ${ }^{1}$. Those under 50 years old should be investigated if iron deficiency recurs within 12 months of iron replacement ${ }^{1}$, or they could be tested using FIT $^{10}$.

In patients with anaemia due to low levels of other haematinics (B12, folate), bidirectional endoscopies are not indicated, however, if pernicious anaemia is diagnosed, gastroscopy may be appropriate.

Dual pathology may be found in $1-10 \%$ of patients ${ }^{1}$, so bi-directional endoscopies should be performed. 
Endoscopic investigations should only be performed if the benefit of the procedure outweighs the risk. Patients with significant comorbidities, in whom the management would not be altered by the result should not undergo endoscopic procedures.

Colonoscopy is preferable to a CT virtual colonoscopy (CTVC) as it can detect superficial lesions, however, a CTVC is a reasonable alternative in some patients as the sensitivity for identifying lesions larger than $10 \mathrm{~mm}$ is over $90 \%{ }^{9}$.

Radiological or endoscopic examination of the small bowel with a CT enterography, small bowel MRI or capsule endoscopy should be considered after negative bidirectional endoscopies if there are symptoms suggestive small bowel disease (e.g. Crohn's disease), such as abdominal pain, weight loss, or raised inflammatory markers. Small bowel capsule endoscopy should be considered if there is evidence of persistent obscure GI bleeding as indicated by a failure to maintain or restore the haemoglobin with iron supplementation ${ }^{13,14}$. If this is normal, non-GI cancer should be investigated for.

\section{References}

1. Goddard AF, James MW, McIntyre AS, et al. Guidelines for the management of iron deficiency anaemia. BSG. 2011.

2. Dahlerup JF, Eivindson M, Jacobsen BA, et al. Diagnosis and treatment of unexplained anaemia with iron deficiency without overt bleeding. Danish Medical Journal. 2014

3. Bouri S, Martin J. Investigation of iron deficiency anaemia . Clin Med. 2018; 18(3): 242-244. doi:10.7861/clinmedicine.18-3-242.

4. Nicholas G, Bennoun M, Devaux I, et al. Lack of Hepcidin gene expression and severe tissue iron overload in upstream stimulatory factor 2 (USF2) knockout mice. Proc Natl Acad Sci. 2001; 98: 8780-8785.

5. Roy C, Mak HH, Akpan I, et al. Hepcidin antimicrobial peptide transgenic mice exhibit features of the anaemia of inflammation. Blood. 2007; 109: 4038-4044.
6. Nemeth E, Rivera S, Gabayan V, et al. IL-6 mediates hypoferremia of inflammation by inducing the synthesis of iron regulatory hormone Hepcidin. J Clin Invest. 2004; 113(9): 1271-1276.

7. Nemeth E, Tuttle MS, Powelson J, et al. Hepcidin regulates cellular iron efflux by binding to ferroportin and inducing its internalization. Science. 2004; 306: 2090-2093.

8. Kroll MH, Jiji V, Jiji R. Microcytic hypochromic anaemia associated with renal cell carcinoma. South Med J. 1984; 77: 635-7.

9. Regge D, Laudi C, Galatola G, et al. Diagnostic accuracy of computed tomographic colonography for the detection of advanced neoplasia in individuals at increased risk of colorectal cancer. JAMA. 2009; 301: 453-61.

10. NHS National Institute for Health and Clinical Excellence. Suspected cancer: recognition and referral. Clinical guidance NG12. 2015. https://www.nice.org.uk/guidance/ng12

11. Hirai HW, Tsoi KK, Chan JY, et al. Systematic review with metaanalysis:fecal occult blood tests show lower colorectal cancer detection rates in the proximal colon in colonoscopy-verified diagnostic studies. Aliment Pharmacol Ther. 2016; 43(7): 755-64.

12. Robertson DJ, Lee JK, Boland CR, et al. Recommendations on fecal immunochemical testing to screen for colorectal neoplasia: a consensus statement by the US Multi-Society Task Force on Colorectal Cancer. Gastroenterology. 2017; 152(5): 1217-1237.

13. Davies GR, Benson MJ, Gertner DJ, et al. Diagnosis and therapeutic push type enteroscopy in clinic use. Gut 2008; 57: 125-36.

14. Sidhu R, Sanders DS, Morris AJ, et al. Guidelines on small bowel enteroscopy and capsule endoscopy in adults. Gut. 2008; 57: 125-36.

15. NHS National Institute for Health and Clinical Excellence. Quantitative faecal immunochemical test to guide referral for colorectal cancer in primary care. Diagnostics guidance DG30. 2017. www.nice.org.uk/ guidance/dg30

16. Cappellini MD, Comin-Colet J, Francisco AD, et al. Iron deficiency across chronic inflammatory conditions: International expert opinion on definition, diagnosis and management. Am J Hematol. 2017; 92(10): 1068-1078.

17. Wagner CV, Stoffel ST, Freeman M, et al. General Practitioners' awareness of the recommendations for faecal immunochemical tests (FITs) for suspected lower gastrointestinal cancers: a national survey. BMJ Open. 2019; 9: e025737. 